\title{
2D NUMERICAL SIMULATION OF TIDAL BORE ON QIANTANG RIVER USING KFVS SCHEME
}

\author{
PAN Cun-Hong ${ }^{1}$ LU Hai-Yan²
}

\begin{abstract}
Qiantang estuary is famous worldwide for tidal bore, whose maximal height reaches about $4 \mathrm{~m}$. In this paper, a new numerical model, based on Boltzmann equation, Kinetic Flux Vector Splitting (KFVS) scheme with the finite-volume method combined with the improved Riemann solution for moving boundary treatment, is constructed to numerically simulate the tidal bore on the Qiantang River. The model is discretized by unstructured triangular grid and has the second order accuracy in spaces. The simulated results show that the model can simulate the whole process of formation, evolution, and dissipation of the tidal bore on the Qiantang River. The typical bore sceneries such as the crossed bore, the thread-shape bore and the returned tidal bore have been reproduced. These studies lead to more understanding about the characteristics of the tidal bore.
\end{abstract}

Keywords: Qiantang estuary; tidal bore; numerical modeling; KFVS scheme; moving boundary

\section{INTRODUCTION}

Propagating into the Hangzhou Bay (Fig.1), the range of the tide wave from the East China Sea increases gradually with the rapid narrowing of the width of the bay. At Ganpu, the end of the bay, the annual-averaged tidal range reaches $5.62 \mathrm{~m}$, with the maximum of $9 \mathrm{~m}$. Caused by the shallow-water effect, nonlinear deformation of the tide wave increases upstream. Finally, the sudden rising of the flooding wave front - the tidal bore - is formed at the upper reach of Ganpu. It becomes stronger upward and reaches the highest at Daquekou. The largest bore can be about $4 \mathrm{~m}$ in height. It decreases further upward, and disappears in the reach from Wenjiayan to Fuyang. The bore-affected reach is about $90 \mathrm{~km}$ long, where propagating rate generally ranges $4-7 \mathrm{~m} / \mathrm{s}$ and the instant rising rate of water level is more than $1 \mathrm{~m} / \mathrm{s}$. The maximum velocity reached $12 \mathrm{~m} / \mathrm{s}$.

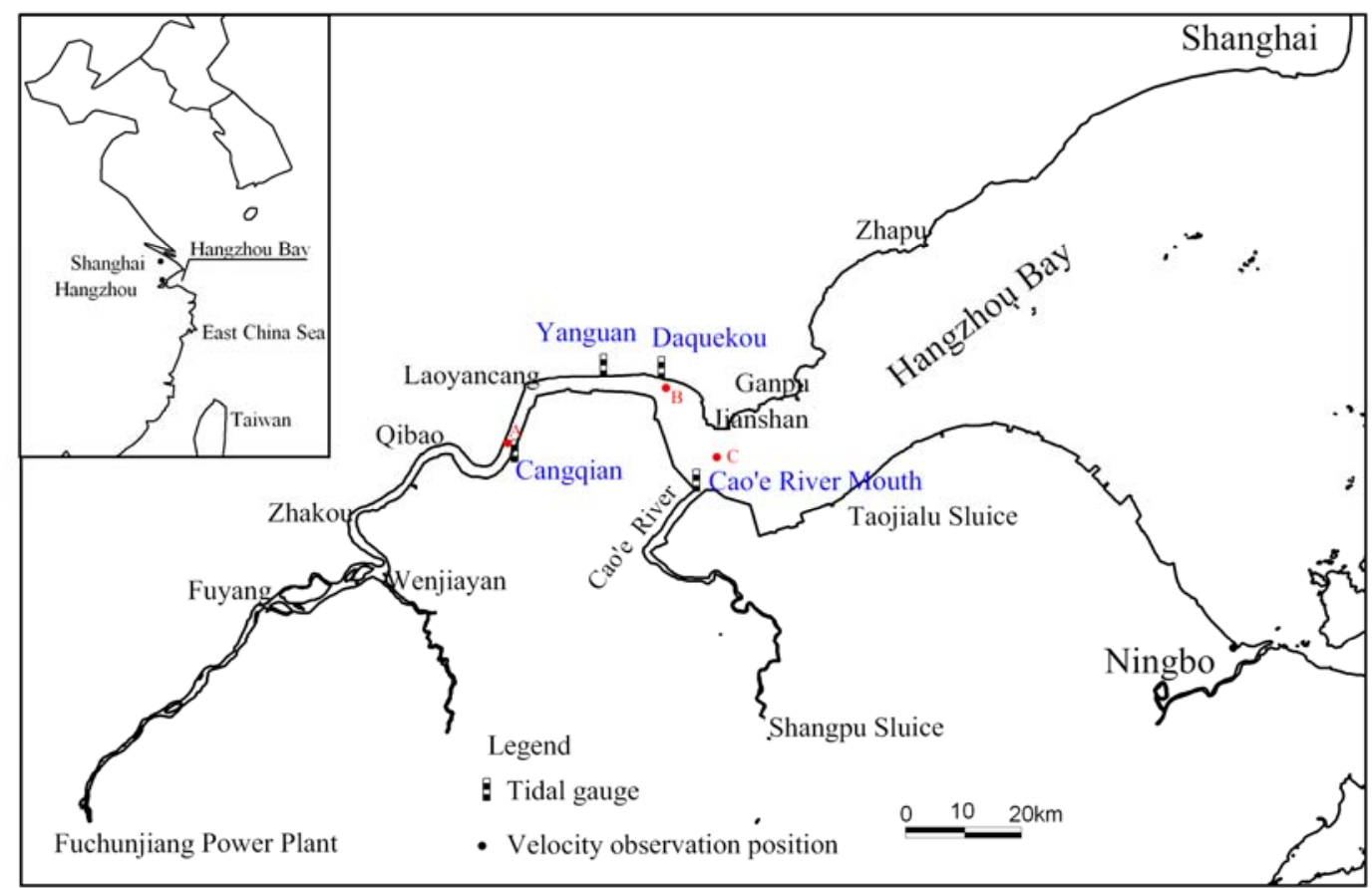

Figure 1. Location of Qiantang River

There exists a sudden variation of the tidal levels, current velocities and water fluxes when the bore passes by, so the tidal bore is a typical discontinuous shallow water flow. When we solve the

\footnotetext{
${ }^{1}$ Professor, Zhejiang Institute of Hydraulics and Estuary, 50 East Fengqi Road Hangzhou 310020, China.

${ }^{2}$ Senior Engineer, Zhejiang Institute of Hydraulics and Estuary, 50 East Fengqi Road Hangzhou 310020, China.
} 
discontinuity problem, traditional numerical schemes frequently do not work. The reason is the traditional schemes are linear form which can not meet the requirements of restraining illusive oscillation and attaining high-resolution synchronously. Subsequently the numerical viscosity of schemes is too large or too small. The former would result in excessive numerical dissipation which will flatten the changing suddenly physical variables and as a result the discontinuity can not be reflected. The latter would lead to illusive oscillation, even numerical instability. Therefore, when we simulate strong discontinuous problems, such as the tidal bore numerically, the numerical scheme should not only be able to simulate large-gradient flow, but also possess high-stability. Since the tidal bore is a strong discontinuity while the governing equations must be shallow water equations in the conservative form, one great difficulty appears for dealing the source term due to bottom topography, namely, the pressure terms at the left side of the equation should always balance the bed slope terms at the right side(Zhou et al., 2001; Hui and Pan, 2003). Furthermore, there are some other difficulties for tidal bore modeling on the Qiantang estuary, such as moving boundary modeling for strong discontinuous shallow water flow, the contradiction between large model domain and small computational cells, etc.

Since the 1960s, the numerical simulations of the tidal bore on the Qiantang estuary have been extensively carried out. Jin et al. (1965), Zhao (1985) solved the 1-D Saint-Venant equations using the method of characteristics and calculated flow variables with the shock-fitting method when the bore passes by. Tan et al. (1995) solved the 2-D shallow water equations using the Osher scheme based on an approximate Riemann solver and simulated the Qiantang bore using the shock-fitting method. Su et al. (2001) solved the 1-D Saint-Venant equations and 2-D shallow water equations using NND scheme, and simulated primarily the formation and evolution of the Qiantang bore with the shock-capturing method. Madsen et al. (2005) simulated the Qiantang bore using improved software MIKE 21. All of the studies help us to understand more characteristics of the tidal bore.

Hui and Pan (2003) proposed Water Level - bottom Topography Formulation (WLTF), and resolved the well-balanced problem for solving 2-D shallow water equations based on quadrilateral cells by incorporating the WLTF method and the discretization techniques of the source terms due to bottom topography. Pan et al. (2007) developed a 2-D numerical model for tidal bore using Godunovtype scheme and structured arbitrary quadrilateral cells. In this paper, a 2-D well-balanced model is developed to simulate the Qiantang bore by solving the shallow water equations with the Kinetic Flux Vector Splitting (KFVS) scheme. The model domain is discretized by an unstructured triangular grid in order to fit the complex local boundaries and bottom topography. It is shown that the model can be used to simulate the formation, evolution, and dissipation of the Qiantang bore.

\section{GOVERNING EQUATIONS AND SOLUTIONS}

A 2-D collisionless Boltzmann equation is (Xu, 1998)

$$
\frac{\partial f}{\partial t}+c_{x} \frac{\partial f}{\partial x}+c_{y} \frac{\partial f}{\partial y}+\phi_{x} \frac{\partial f}{\partial c_{x}}+\phi_{y} \frac{\partial f}{\partial c_{y}}=0
$$

in which, $f$ represents the probability distribution function of the particle velocities; $c_{x}$ and $c_{y}$ represent the particle velocities in $x$ - and $y$-direction, respectively; $\phi$ represents external force, including frictional force term and the gravitational force term acting on the particle due to the uneven riverbed, $\phi_{x}=g\left(S_{0 x}-S_{f x}\right) ; \phi_{y}=g\left(S_{0 y}-S_{f y}\right) ; g$ is the acceleration due to gravity; $S_{f x}$ and $S_{f y}$ are the frictions in the $x$ - and $y$-direction, respectively; $S_{0 x}$ and $S_{0 y}$ are the source terms due to bottom topography in the $x$ - and $y$-direction, respectively; $S_{0 x}=-\partial b / \partial x, S_{0 y}=-\partial b / \partial y, b$ is the bottom elevation.

$f$ is a function of spaces $x$ and $y$, time $t$, and the particle velocities $c_{x}$ and $c_{y}$. Its distribution function under equilibrium, herein dented by $q$, obeys the Maxwellian distribution. Then the 2-D shallow water equations could be obtained by taking into account the relationship between the Boltzmann variables and the macroscopic variables (Deng and Ghidaoui, 2001),

$$
\int_{-\infty}^{+\infty} \int_{-\infty}^{+\infty} q d c_{x} d c_{y}=h \quad \int_{-\infty}^{+\infty} \int_{-\infty}^{+\infty} q c_{x} d c_{x} d c_{y}=h u
$$




$$
\begin{aligned}
& \int_{-\infty}^{+\infty} \int_{-\infty}^{+\infty} q c_{y} d c_{x} d c_{y}=h v \quad \int_{-\infty}^{+\infty} \int_{-\infty}^{+\infty} q c_{x} c_{y} d c_{x} d c_{y}=h u v \\
& \int_{-\infty}^{+\infty} \int_{-\infty}^{+\infty} q c_{x}^{2} d c_{x} d c_{y}=h u^{2}+\frac{1}{2} g h^{2}
\end{aligned}
$$

The model method based on Boltzmann equation does not directly solve the shallow water equations, but indirectly through Boltzmann Eq. (1).

Eq. (1) can be multiplied by $\left(1, c_{x}, c_{y}\right)^{T}$, and integrated the particle velocity over the space, then

$$
\frac{\partial \boldsymbol{E}}{\partial t}+\frac{\partial \boldsymbol{F}}{\partial x}+\frac{\partial \boldsymbol{G}}{\partial y}=\boldsymbol{S}
$$

where

$$
\begin{aligned}
& \boldsymbol{E}=\left[\begin{array}{lll}
h, & h u, & h v
\end{array}\right]^{T} \\
& \boldsymbol{F}=\left[\int_{-\infty}^{+\infty} \int_{-\infty}^{+\infty} c_{x} f d c_{x} d c_{y}, \quad \int_{-\infty}^{+\infty} \int_{-\infty}^{+\infty} c_{x}^{2} f d c_{x} d c_{y}, \int_{-\infty}^{+\infty} \int_{-\infty}^{+\infty} c_{x} c_{y} u v f d c_{x} d c_{y}\right]^{T} \\
& \boldsymbol{G}=\left[\int_{-\infty}^{+\infty} \int_{-\infty}^{+\infty} c_{y} f d c_{x} d c_{y}, \quad \int_{-\infty}^{+\infty} \int_{-\infty}^{+\infty} c_{x} c_{y} f d c_{x} d c_{y}, \quad \int_{-\infty}^{+\infty} \int_{-\infty}^{+\infty} c_{y}^{2} f d c_{x} d c_{y}\right]^{T} \\
& \boldsymbol{S}=\left[0, \quad g h\left(S_{0 x}-S_{f x}\right), \quad g h\left(S_{0 y}-S_{f y}\right)\right]^{T}
\end{aligned}
$$

in which $h$ is water depth; $u$ and $v$ are velocity components in the $x$ - and $y$-directions.

The Boltzmann-equation-based scheme solves Eq. (2) to get the macroscopic flow variables. In this paper, the model domain is subdivided into a set of arbitrary triangular cells to simulate natural waters with complex plane configurations. The cells are set to be control volumes and the physical variables are set in the centroids of the cells. Let $\Omega_{i}$ be the ith cell and $\Gamma$ be its boundary, then Eq. (2) can be discretized by the finite-volume method incorporating with Green's theorem as follows:

$$
A_{i} \frac{\partial \boldsymbol{E}}{\partial t}+\oint_{\Gamma}(\boldsymbol{F} \cos \theta+\boldsymbol{G} \sin \theta) d l=\iint_{\Omega_{i}} \boldsymbol{S}_{i} d x d y
$$

where $A_{i}$ is the area of cell $\Omega_{i} ;(\cos \theta, \sin \theta)$ are the unit normal vector quantities that point outward $\Gamma$; $d l$ is the line integral of infinitesimal. If $\boldsymbol{F} \cos \theta+\boldsymbol{G} \sin \theta$ is denoted by $\boldsymbol{F}_{n}$ and the time derivative in Eq.(7) is descretized with the forward difference method, then the numerical solution based on finite-volume method is

$$
\boldsymbol{E}_{i}^{n+1}=\boldsymbol{E}_{i}^{n}-\frac{\Delta t}{A_{i}} \sum_{j=1}^{3} \boldsymbol{F}_{n j} l_{j}+\frac{\Delta t}{A_{i}} \iint_{\Omega_{i}} \boldsymbol{S}_{0 i} d x d y+\Delta t \boldsymbol{S}_{f i}
$$

in which, $\Delta t$ is time step; subscript $j$ presents the $j$ th side of the ith cell; $l_{j}$ is the length of the sides of the cell; superscript $n$ is the number of time step.

The core of solving Eq. (8) is calculation of the numerical fluxes through cell interfaces and treatment of the source terms due to bottom topography. Herein the fluxes at interfaces are solved by KFVS scheme (Pan and $\mathrm{Xu}, 2006$ ), and all other terms are solved using the same approach of the normal finite volume method. Since the governing equations are written in the conservative form, the pressure term at the left side should balance the source term due to bottom topography at the right side. The treatment for this problem has been described in detail in Pan and Xu (2006) in which the scheme has second order accuracy in space. Several numerical tests showed that KFVS scheme could capture strong discontinuity and possesses good stability and high-solution (Pan and $\mathrm{Xu}, 2006$ ).

\section{MOVING BOUNDARY TECHNIQUES}

Moving boundary is one of the difficulties for tidal bore modeling. The tidal bore arrives at low tide and extensive tidal flats expose. Therefore, whether the moving boundary could be dealt with 
accurately influences the model results to a large extent, even leads to instability of calculation. In the last twenty years, many scholars proposed various methods for moving boundary handling. However, most were not applicable to the wet/dry boundary of discontinuity shallow water flow, but moving boundary occurs at the same time when tidal bore arrives. Recently, the wetting/drying techniques based on approximate Riemann solver have been applied for the moving boundary treatment of discontinuity flow (Sleigh et al, 1998; Brufau et al., 2002, 2004). In this contribution, the exact Riemann solution for dry bed of discontinuity flow is developed for the wetting/drying handling.

If local coordinate system is introduced and the common side of two adjacent triangular cells is set to be the origin (denoted $x$ for $x$ simplification), then the Riemann problem of 2-D shallow water flow is as follows

where

$$
\left\{\begin{array}{c}
\frac{\partial M}{\partial t}+\frac{\partial N}{\partial x}=0 \quad t>0 \\
M(0, x)= \begin{cases}M_{L}, & x<0 \\
M_{R}, & x>0\end{cases}
\end{array}\right.
$$

$$
M=\left[\begin{array}{c}
h \\
h \omega \\
h \tau
\end{array}\right] \quad N=\left[\begin{array}{c}
h \\
h \omega^{2}+\frac{1}{2} g h^{2} \\
h \omega \tau
\end{array}\right]
$$

Subscripts $\mathrm{L}$ and $\mathrm{R}$ denote the left and the right of the common side, respectively; $\omega$ is normal velocity; $\tau$ is tangential velocity.

Assuming $W$ being wet bed and $W_{0}$ being dry bed (moving boundary)

$$
W=\left[\begin{array}{l}
h \\
\omega \\
\tau
\end{array}\right] \quad W_{0}=\left[\begin{array}{l}
h_{0} \\
\omega_{0} \\
\tau_{0}
\end{array}\right]
$$

where $h_{0}$ is zero, $\omega_{0}$ and $\tau_{0}$ are arbitrary values.

For dry bed, there exist three cases, i.e., "the dry bed is at the right", "the dry bed is at the left" and "the dry bed is at the middle" (Toro, 2001). Considering that the probability for the third case is very small while the second is similar to the first, the authors just describe the first case as follows.

For the case of the dry bed at the right, the initial data of the Riemann problem is

$$
W(0, x)=\left\{\begin{array}{cc}
W_{L} \neq W_{0} & x<0 \\
W_{0}(\text { dry bed }) & x>0
\end{array}\right.
$$

The structure of Riemann solution is shown in Fig. 2. In this figure $a=\sqrt{g h}$. The solutions contains a left rarefaction wave associated with the eigenvalue $\lambda=\omega-a$, and a contact discontinuity of speed $S_{*_{L}}$, i.e., wet/dry interface which coincides with the tail of the rarefaction (see dashed line on the right-hand side of Fig.2). The solution of the Riemann problem is

$$
W_{L 0}(t, x)=\left\{\begin{array}{cc}
W_{L} & x / t \leq \omega_{L}-a_{L} \\
W_{L f a n} & \omega_{L}-a_{L} \leq x / t \leq S_{* L} \\
W_{0} & S_{*_{L}} \leq x / t
\end{array}\right.
$$

where 


$$
\begin{aligned}
& W_{L f a n}=\left\{\begin{array}{c}
a=\frac{1}{3}\left(\omega_{L}+2 a_{L}-\frac{x}{t}\right) \\
\omega=\frac{1}{3}\left(\omega_{L}+2 a_{L}+\frac{2 x}{t}\right) \\
\tau=\tau_{L}
\end{array}\right. \\
& S_{* L}=\omega_{L}+2 a_{L}
\end{aligned}
$$

While $h_{L}$ is water depth of the left wet cell, Eq.(10) and (11) only are appropriate for flat bottom. In fact, according to WLTF idea (Hui and Pan 2003), set $h_{L}=Z_{L}-b_{L R}$, Eq.(10) and (11) can be extended to uneven bottom, in which, $Z_{L}$ is water level of the left wet cell, and $b_{L R}$ is bottom elevation at the wet/dry interface.

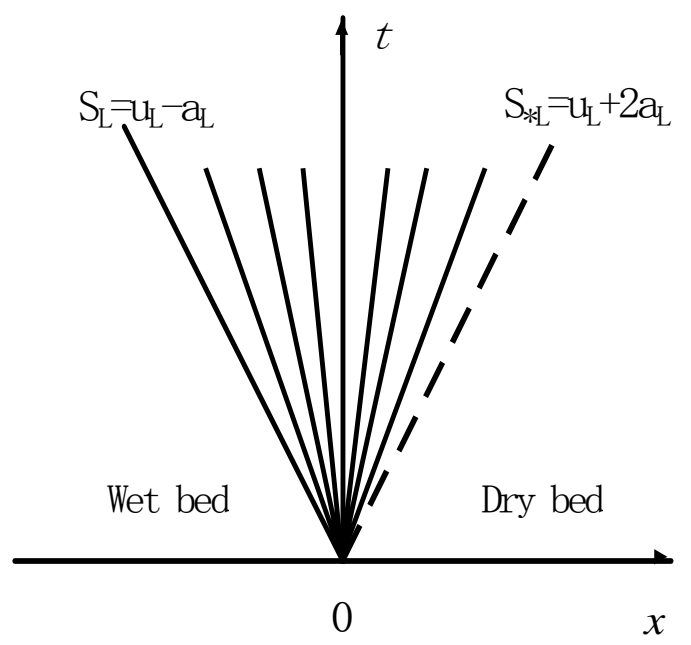

Figure 2. The wave structure of Riemann solution on right dry bed

\section{MODEL RESULTS}

\section{Computational Conditions}

The downstream open boundary is set at the mouth of Hangzhou Bay, the upstream open boundaries of the Qiantang River and Cao'e River are set at the Fuchunjiang power station and Shangpu sluice, respectively, as shown in Fig.1. The computational region was discretized by a triangular grid, composed of 69062 elements and 36065 nodes.

The upstream boundary conditions were specified by observed water fluxes and the downstream boundary condition was specified by observed tidal level data. The current velocities at downstream boundary were calculated by 1-D characteristic difference scheme (Pan et al., 2007). On the solid-wall boundary, the slip condition was employed and the normal velocity was set to be zero. The interface flux on the solid boundary was solved by using the approach of image reflection, which may be done by employing a "ghost" cell outside the domain that is symmetric to the cell inside the domain.

The model validations were carried out with observed hydrological data during September, 2000 and October, 2007. After repetitious tests, the computed results agree well with the measured data when the Manning coefficients ranged from 0.004 to 0.008 for flood tide and from 0.006 to 0.013 for ebb tide in the model.

\section{Model Results}

- $\quad$ Tidal Level and Velocity Validations. 
In the measurements during September, 2000 and October, 2007, observed data from 12 tidal gauges were used for model validation. The comparison between computed and observed tidal levels indicates a good agreement with each other for high and low levels, the ranges and phases of tides. Fig. 3 shows the comparison between computed and observed tidal levels at the mouth of Cao'e River, Daquekou, Yanguan and Cangqian (see Fig. 1 for locations) during September, 2000. Unfortunately, observed data of current velocity were unavailable during September, 2000, and hence the velocity was validated using observed data during October, 2007. Fig. 4 shows model validation of current velocities and directions at monitoring stations A, B and C during October, 2007, which indicates a good agreement between calculations and observations.
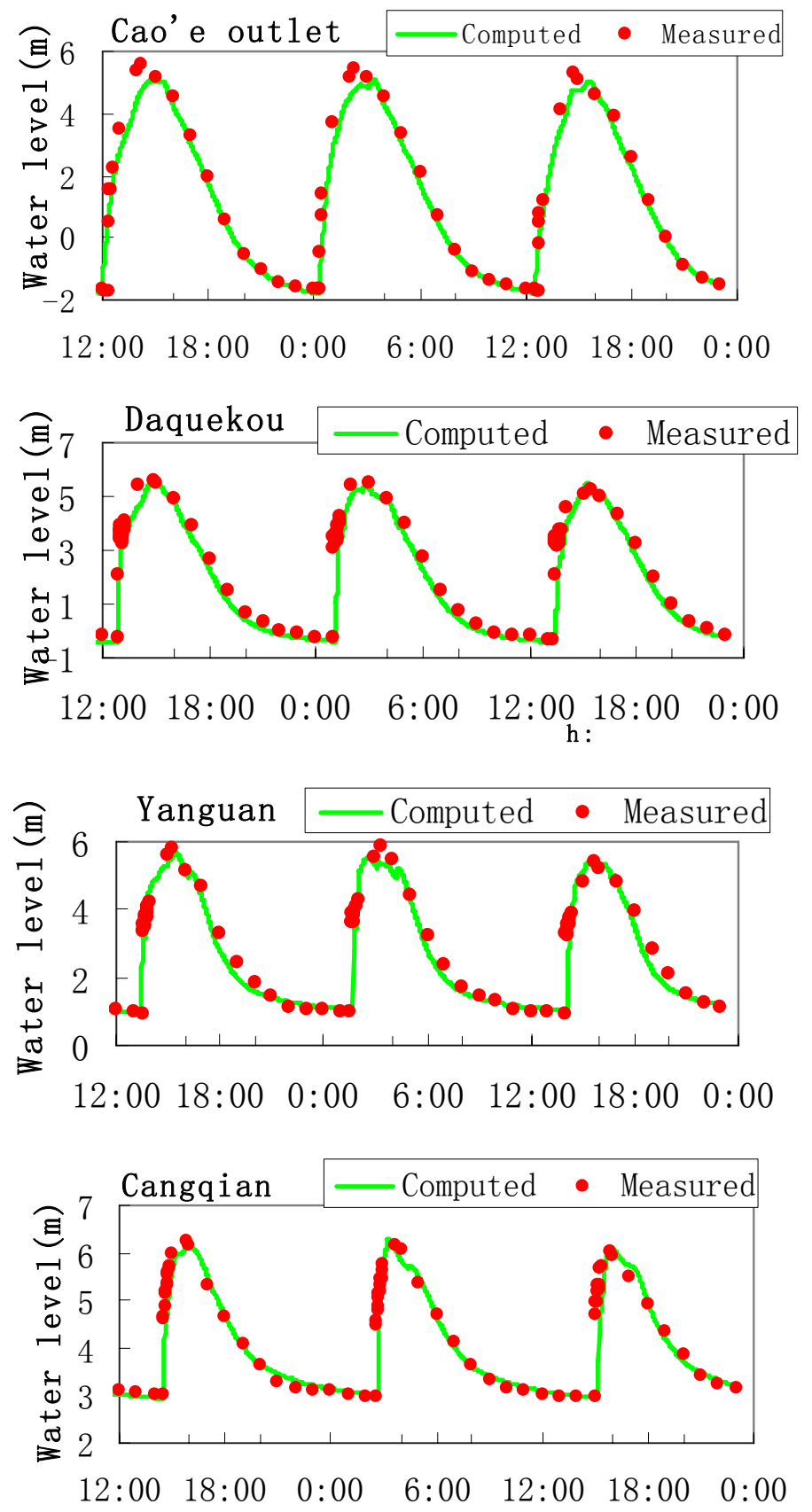

Figure 3. Comparison of the tidal level between computed and measured 

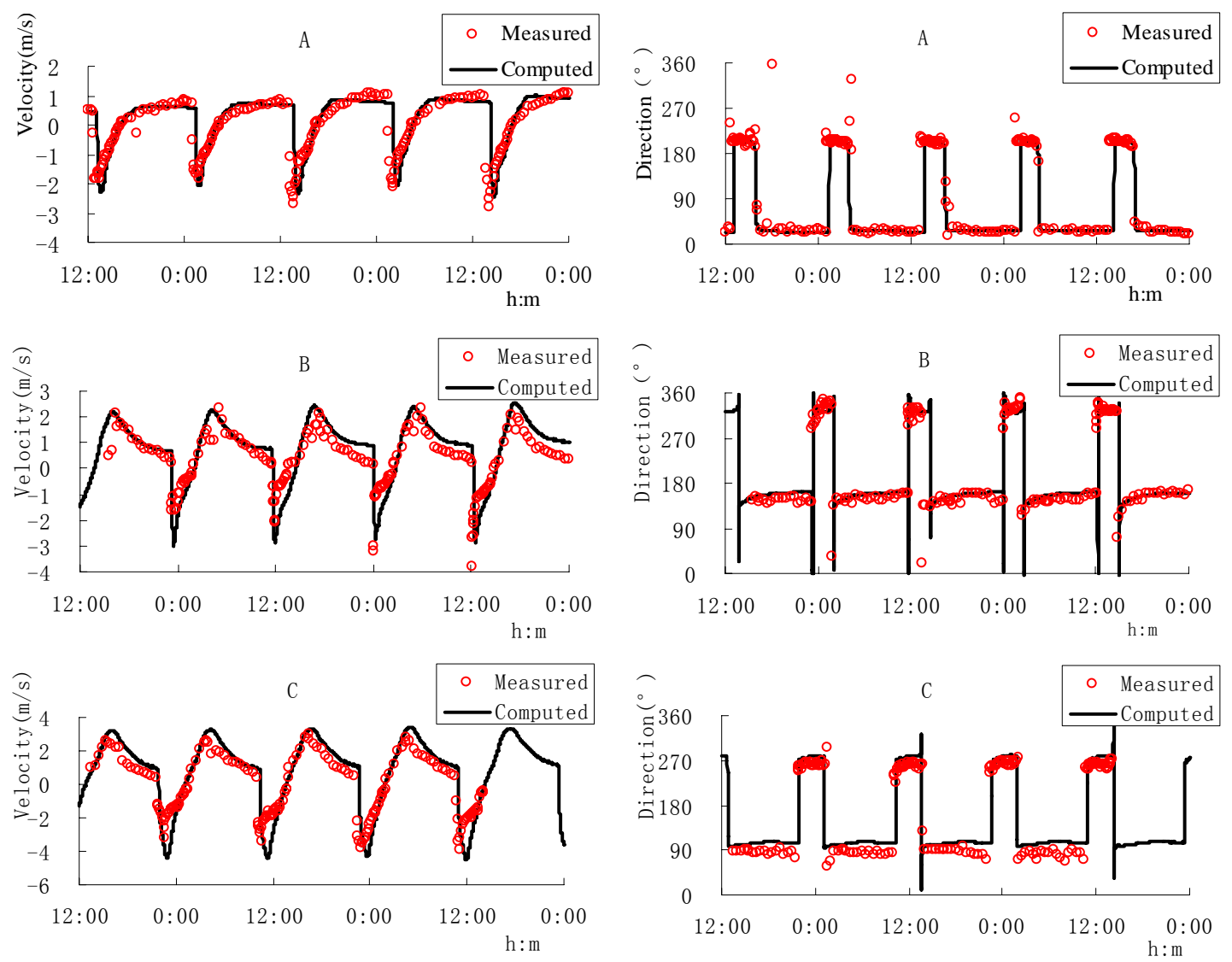

Figure 4. Comparison of the velocity and its direction between computed and measured

- Heights of the Bore.

Actually, the tidal bore is formed gradually, namely, the rising rate of the flood tide front increases gradually. It is considered as tidal bore, once the discontinuity of tidal levels of the flood tide front could be distinguished by naked eyes. Although the model could reproduce the formation of the bore, it is difficult to judge the initial site of the bore because the grid around the initial site is too coarse, about $750 \mathrm{~m}$, and tidal level jump is not distinguishable at the initial stage of the bore formation.

Fig.5 shows the comparison between calculated and observed heights of the bore along the river. They are basically consistent with each other, although the calculated are a little less than the observed. Fig. 6 shows the calculated and observed processes of the bore at Yanguan gauge. The model results could reflect the rapid rise of tidal levels when the bore arrives, and this is denoted by the discontinuity in this figure.

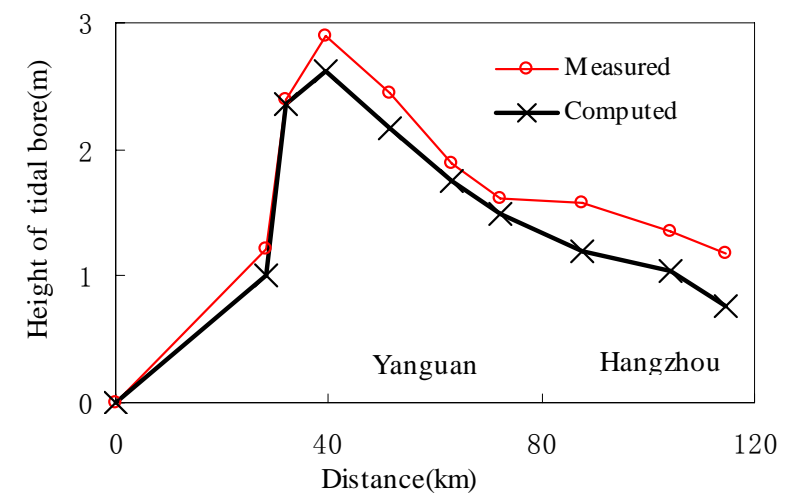

Figure 5. Comparison of the height of the tidal bore between computed and measured 


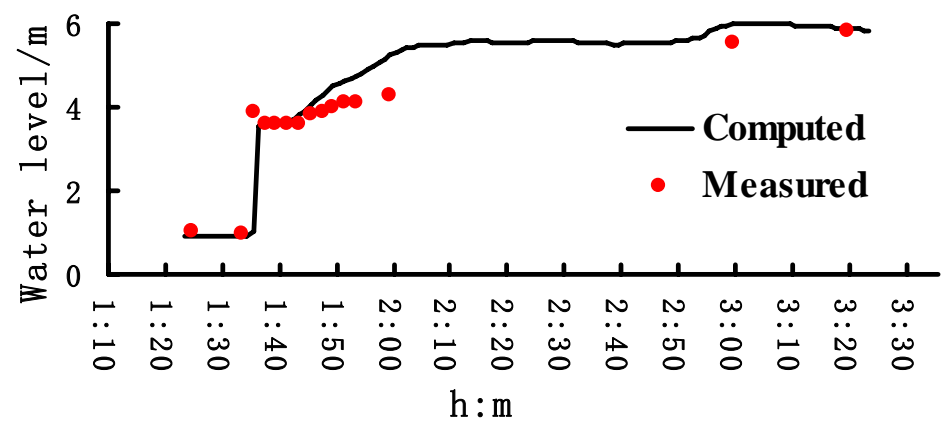

Figure 6. Comparison of the process of the tidal bore between computed and measured at Yanguan

- $\quad$ Propagation Speed of the Bore.

Table 1 illustrates the comparison between the computed and observed propagation speeds of the bore and the time when the bore arrives at representative stations. The calculated times for the bore arrival are close to the observed ones, with the maximal error being 6 minutes. The observed propagation speed ranges between $4.96 \mathrm{~m} / \mathrm{s}$ and $8.03 \mathrm{~m} / \mathrm{s}$.The calculated propagation speeds agree well with the observed values. The propagation speeds of the bore from Qibao to Wenjiayan are relatively large, this is mainly due to the large water depths at this reach.

\begin{tabular}{|c|c|c|c|c|c|c|c|c|}
\hline Stations & Jianshan & Daquekou & Yanguan & Cangqian & Qibao & & Zhakou & Wenjiayan \\
\hline $\begin{array}{l}\text { observed time for bore } \\
\text { arrival }(h: m)\end{array}$ & $0: 20$ & $0: 58$ & $1: 34$ & $2: 35$ & $3: 19$ & & $3: 53$ & $4: 15$ \\
\hline $\begin{array}{l}\text { calculated time for bore } \\
\text { arrival (h:m) }\end{array}$ & $0: 23$ & $1: 04$ & $1: 39$ & $2: 35$ & $3: 16$ & & $3: 54$ & $4: 19$ \\
\hline absolute error(h:m) & 0:03 & 0:06 & 0:05 & $0: 00$ & $-0: 03$ & & $0: 01$ & 0:04 \\
\hline distance $(\mathrm{km})$ & 11.3 & 11.2 & 21.0 & 16.0 & & 16.0 & & 10.6 \\
\hline $\begin{array}{l}\text { observed propagation } \\
\text { speed }(\mathrm{m} / \mathrm{s})\end{array}$ & 4.96 & 5.19 & 5.74 & 6.06 & & 7.84 & & 8.03 \\
\hline $\begin{array}{l}\text { calculated propagation } \\
\text { speed }(\mathrm{m} / \mathrm{s})\end{array}$ & 4.59 & 5.33 & 6.25 & 6.50 & & 7.02 & & 7.07 \\
\hline relative error (\%) & -7 & 3 & 9 & 7 & & -10 & & -12 \\
\hline
\end{tabular}

- $\quad$ Current Velocity of the Bore.

In the reach where the bore occurs, current velocities reach their maximal values normally after several minutes or dozens of minutes of the bore-passing-by. The former and latter time span correspond to the bore passing by the upstream and the downstream of the river, respectively.From the downstream to the upstream, the maximal velocity varies increasingly at first and then decreasingly, with the extreme velocity appearing at Yanguan reach (Pan et al., 2007).

Relatively large velocity appears at the convex bank of bend in transversal. In the computational region, there are many bends such as Daquekou, Laoyancang, Zheshanwan, Qibao, Zhakou, and so on. At all these bends maximal velocities at the convex banks are larger than those at the concave banks. Fig. 7 shows the computational contours of maximal velocity at Laoyancang, where the maximal velocity at the convex bank could be more than $7 \mathrm{~m} / \mathrm{s}$ while that at concave bank is only $2 \sim 3 \mathrm{~m} / \mathrm{s}$. This scenario is different from the normal velocity distributions of rivers. The reason could be interpreted as follows.

According to 1-D continuity equation and momentum equation, and if the current velocity before the bore arrives, i.e., at the upstream of the bore, is set to be zero, then

$$
u_{d}=\sqrt{\frac{g}{2}\left(\frac{1}{h_{u}}+\frac{1}{h_{d}}\right)} \Delta h=\sqrt{\frac{g}{2}\left(\frac{1}{h_{u}}+\frac{1}{h_{u}+\Delta h}\right)} \Delta h
$$

where subscript $u$ and $d$ denote the upstream and the downstream of the bore. And other variables are kept the same as the above-mentioned. 
Eq. (12) implies that, if the heights of the bore are the same, then the more shallow the water depth before the bore arrival is, the larger the flow velocity after the bore passes by is. That means, relatively large velocity tends to appear at shallow regions where the bore passes by.

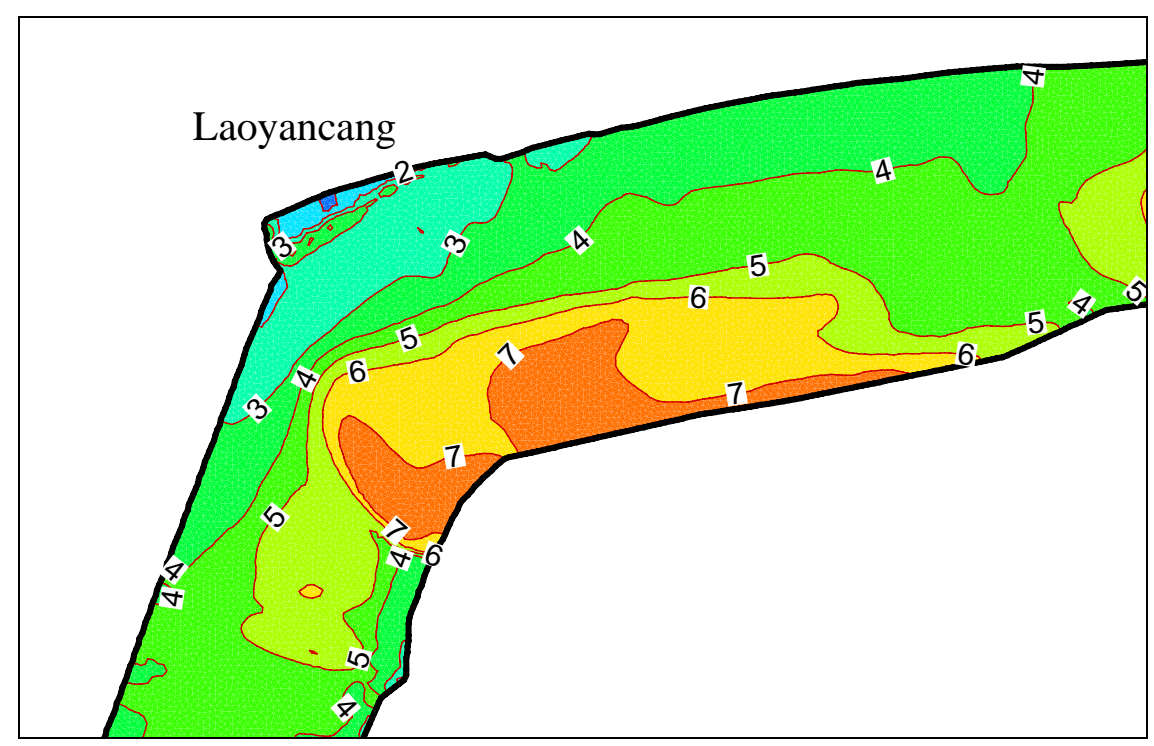

Figure 7. Computational contours of maximum velocity at Laoyancang (unit:m/s)

Fig. 8 shows the evolution of Froude number $F_{r}=\frac{u_{d}}{\sqrt{g h_{d}}}$ in the main channel of Yanguan section. In the figure, flow type changes from subcritical flow to supercritical flow at first, and then from supercritical flow to subcritical flow. Obviously, the variation of the flow type is different from that of steady flows.

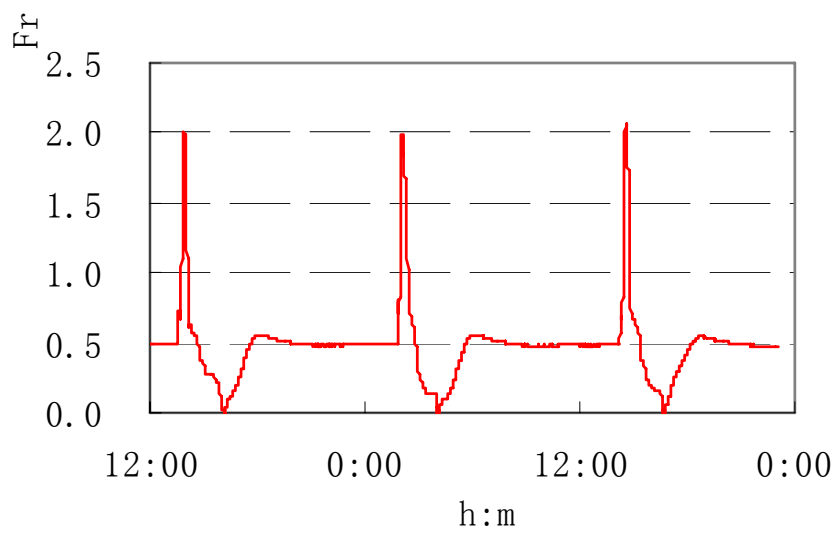

Figure 8. Froude number evolution in the main channel of Yanguan section

- Bore Scenarios.

During propagation of the tidal bore, a series of diverse tidal scenarios are formed, influenced by bathymetry and boundaries.

Crossed tidal bore: In the reach of Jianshan, thanks to the furcated bathymetry, the crossed tidal bore could frequently be formed. Since the height of the crossed bore is small, normally around $1 \mathrm{~m}$, but the model grid is coarse, the observed grand vision of the two fronts intersect at the middle is difficult to be reproduced using the numerical model. Fig. 9 illustrates the distribution of velocity vectors when two fronts intersect.

Thread-shape bore: When the bore propagates upstream, the thread-shape bore is often observed in 
the reach of Yanguan and upper reach of Cangqian. This scenario could be reproduced easily by the model. Fig. 10 is a photo of the thread-shape bore at Yanguan and Fig. 11 shows velocity vectors and tidal level contours of the thread-shape bore at Yanguan.

Returned tidal bore: Returned tidal bore occurs at several spots, among which Laoyancang is the most famous. Fig. 12a and 12b show tidal level contour and synchronous distribution of velocity vectors when the bore appears at Laoyancang, respectively.

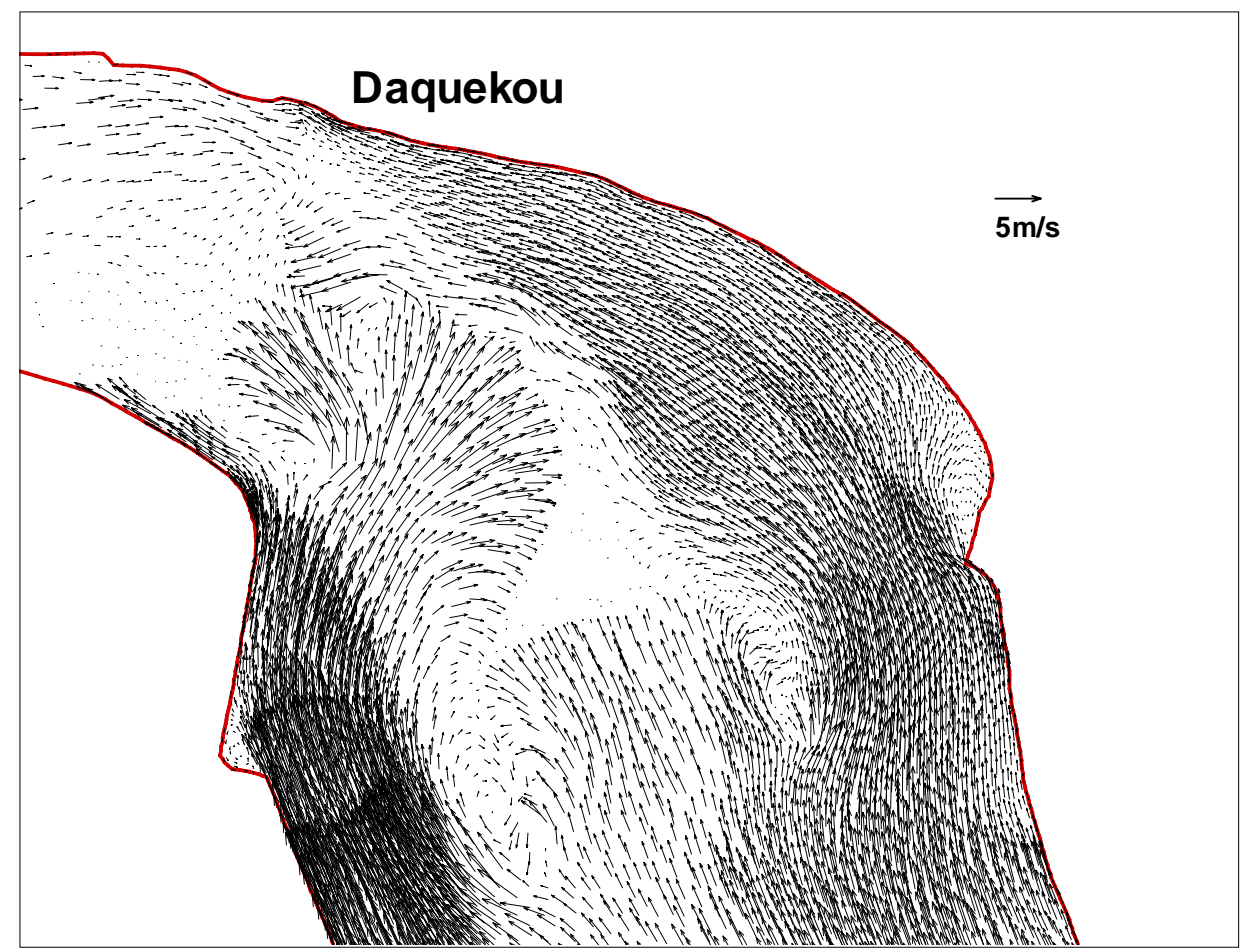

Figure 9. Distribution of velocity vectors when two bores intersection

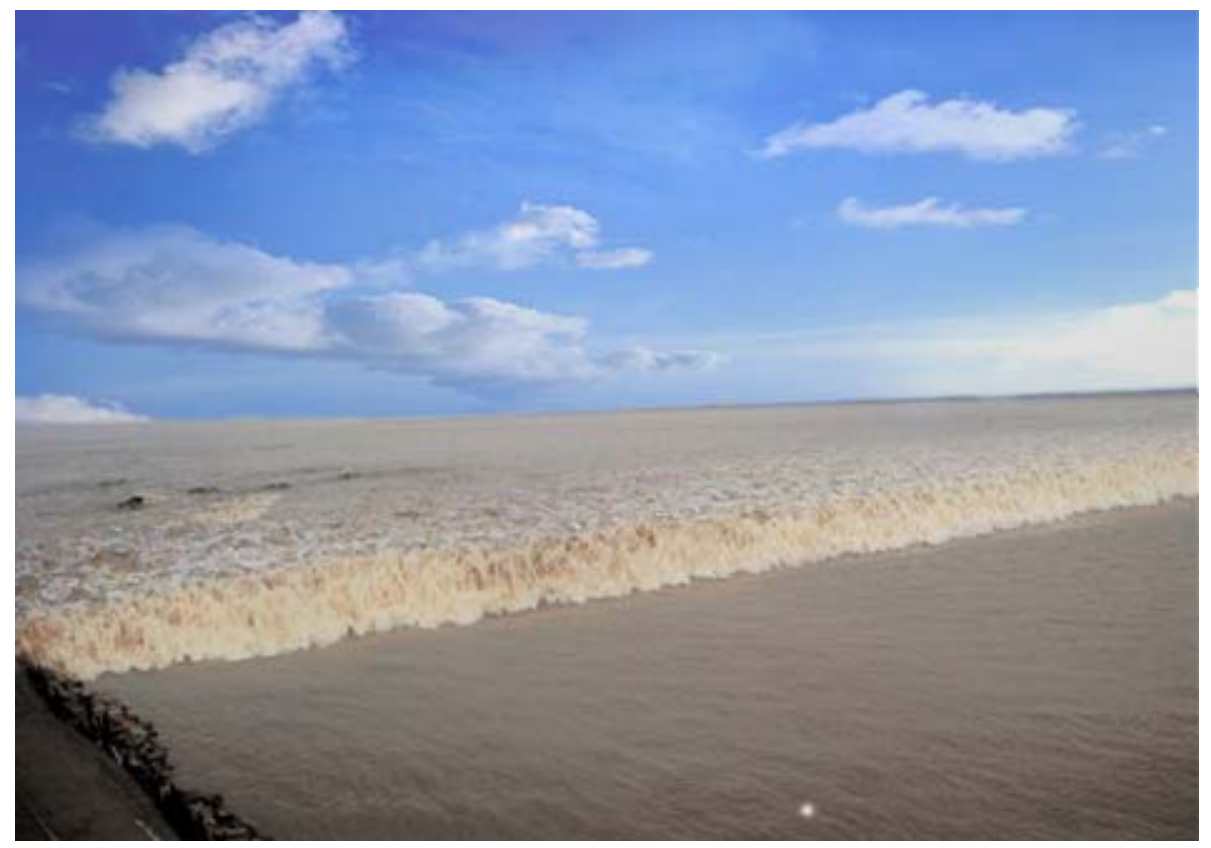

Figure 10. Photo of thread-shape bore at Yanguan 


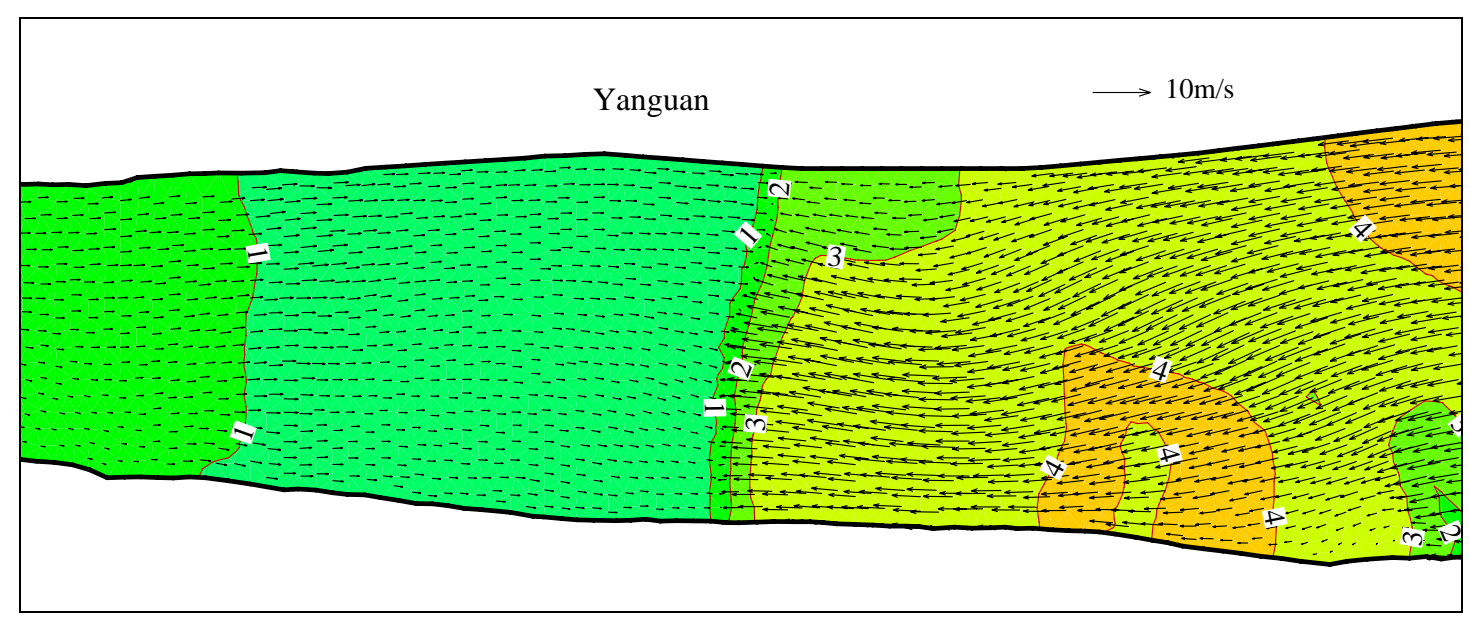

Figure 11. Velocity vectors and tidal level contour of thread-shape bore at Yanguan (unit:m)
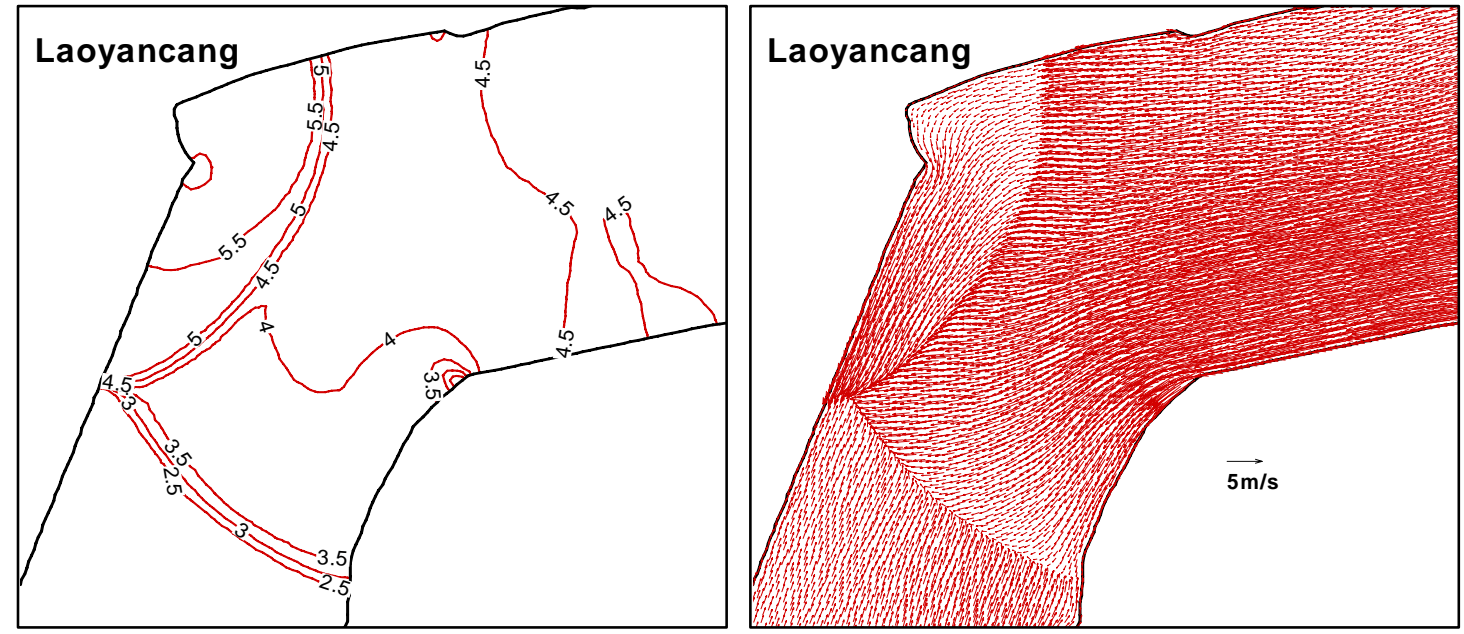

Figure 12a. Tidal level contour when the bore appears at Laoyancang (unit:m)

Figure 12b. Distribution of velocity vectors when the bore appears at Laoyancang

\section{CONCLUSIONS}

After several years of efforts, we have built 2-D numerical model of the tidal bore based on finitevolume method and triangular grid, and solved related critical technical problems such as strong discontinuity, "well-balanced" scheme and moving boundaries, etc. The model simulated the whole process of formation, development and dissipation of the tidal bore on the Qiantang River and reproduced bore scenarios like the crossed bore, the thread-shape bore and the returned bore. The understanding of the characteristics of tidal bore has been improved through analysis of the model results.

Numerical experiments showed that, the numerical model in this study has the advantages of good stability, high-resolution, good conservation and high capacity to fit boundary and bathymetry. Up to now, the 2-D model has been applied to engineering projects such as computation of the tidal bore under the regulation projects in Hangzhou Bay (Pan and Lu, 2009), impact on the tidal bore of estuarine constricting works (Lu et al., 2008) and bridge engineering (Lu et al., 2009). Furthermore, the model has been extended to simulate dam-break flow (Pan et al., 2010) and tsunami.

\section{ACKNOWLEDGMENTS}

The study was partially supported by the National Science Foundation of China (10772163), Water pollution control and management technology major project (2009ZX07424-001), and Zhejiang Provincial Natural Science Foundation of China (Y5080219). 


\section{REFERENCES}

Brufau, P., Vazquez-Cendon M.E., and Garca-Navarro P., 2002. A numerical model for the flooding and drying of irregular domains. International Journal for Numerical Methods in Fluids, 39, 247275.

Brufau, P., Garca-Navarro P., and Vazquez-Cendon M.E., 2004. Zero mass error using unsteady wetting-drying conditions in shallow flows over dry irregular topography. International Journal for Numerical Methods in Fluids, 45, 1047-1082.

Deng, J. Q., and Ghidaoui, M. S. 2001. A Boltzmann based mesoscopic model for contaminant transport in flow system. Advances in Water Resources, 24, 531-550.

Hui, W.H., and Pan Cun-hong. 2003. Water level-bottom topography formulation for the shallowwater flow with application to the tidal bores on the Qiantang River. Computational Fluid Dynamics Journal, 12, 549-554.

Jin, D.H., Liu, G.J., and Zhou, B.H. 1965. 1-D numerical model on tidal bore. Applied Mathematics and Computational Mathematics, 3, 183-195 (in Chinese with English Abstract).

Lu Hai-yan, Pan Cun-hong, Lu Xiang-Xing, and Zeng Jian. 2008. Numerical simulations of the third Haining reclamation Project effete on the tidal bore in the Qiantangn River. Chinese Journal of Hydrodynamics, 23(5): 484-491. (in Chinese with English Abstract)

Lu Hai-yan, Pan Cun-hong, Zeng Jian. 2009.Numerical simulation and analysis for combinational effects of two bridges on the tidal bore in the Qiantang River. The proceedings of the 5th International Conference on Asian and Pacific Coasts, Singapore, Vol.3, 325-333

Madsen, P.A., Simonsen H.J., and Pan Cun-hong. 2005. Numerical simulation of tidal bores and hydraulic jumps. Coastal Engineering, 52, 409-433

Pan Cun-hong, Lin Bing-Yao, and Mao Xian-Zhong. 2007. Case study: numerical modeling of the tidal bore on the Qiantang River, China. Journal of Hydraulic Engineering, 133, 130-138.

Pan Cun-hong, and Xu Kun. 2006. Kinetic flux vector splitting scheme for solving 2-D shallow water equations with triangular mesh. Journal of Hydraulic Engineering, 37, 858-864 (in Chinese with English Abstract).

Pan Cun-hong, and Lu Hai-yan. 2009. 2D numerical model for discontinuous shallow water flows and application to simulation of tidal bore. Journal of Zhejiang University (Engineering Science), 43(11): 2107-2113. (in Chinese with English Abstract)

Pan Cun-hong, Lu Hai-yan, and Zheng Jun, 2010. Two-dimensional numerical model of dam-break flow and its application. Journal of Hydroelectric Engineering, 29(4): 94-100. (in Chinese with English Abstract)

Sleigh, P.A., Gaskell P.H., and Berzins M. 1998. An unstructured finite-volume algorithm for predicting flow in rivers and estuaries. Computers \& Fluids, 27, 479-508.

Su, M. D., Xu X., Zhu J.L., and Hon Y.C. 2001. Numerical simulation of tidal bore in Hangzhou Gulf and Qiantangjiang. International journal for numerical methods in fluids, 36, 205-247.

Tan, W. Y., Hu S.Y., and Han Z.C. 1995. Two-Dimensional numerical modeling of bores in the Qiangtang Estuary. Advances in Water Sciences, 6, 83-93 (in Chinese with English Abstract).

Toro, E.F. 2001. Shock-capturing methods for free-surface shallow flows. John Wiley \& Sons,Chichester.

Xu K 1998. Gas-kinetic scheme for unsteady compressible flow simulations. 29th Computational Fluid Dynamics, Von Karman Institute for Fluid Dynamics Lecture Series. 
Zhou J.G., D.M. Causon, and C.G. Minghamet. 2001. The surface gradient method for the treatment of source terms in the shallow-water equations. Journal of Computational Physics, 168, 1-25.

Zhao X.H. 1985. 1D Mathematical model of the tidal bore on the Qiantang River. Journal of Hydraulic Engineering, 16, 50-54 (in Chinese with English Abstract). 